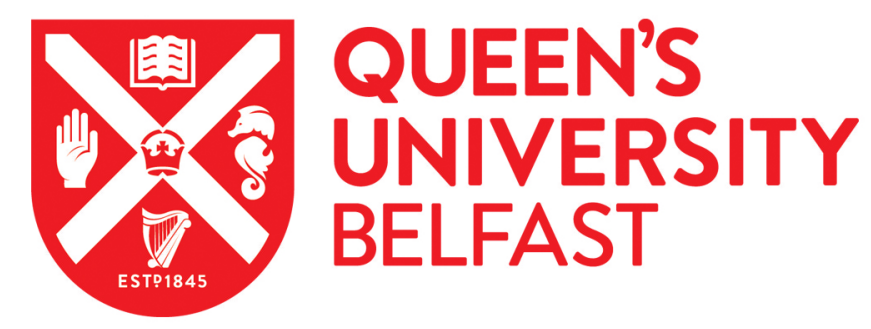

\title{
Alzheimer's disease-like pathology has transient effects on the brain and blood metabolome
}

Pan, X., Bin Nasaruddin, M., Elliott, C. T., McGuinness, B., Passmore, A. P., Kehoe, P. G., Holscher, C., McClean, P. L., Graham, S. F., \& Green, B. D. (2016). Alzheimer's disease-like pathology has transient effects on the brain and blood metabolome. Neurobiology of Aging, 38, 151-163.

https://doi.org/10.1016/j.neurobiolaging.2015.11.014

Published in:

Neurobiology of Aging

Document Version:

Peer reviewed version

Queen's University Belfast - Research Portal:

Link to publication record in Queen's University Belfast Research Portal

Publisher rights

(c) 2016 Elsevier Ltd. This manuscript version is made available under the CC-BY-NC-ND 4.0 license http://creativecommons.org/licenses/bync-nd/4.0/which permits unrestricted use, distribution and reproduction in any medium, provided the author and source are cited.

\section{General rights}

Copyright for the publications made accessible via the Queen's University Belfast Research Portal is retained by the author(s) and / or other copyright owners and it is a condition of accessing these publications that users recognise and abide by the legal requirements associated with these rights.

Take down policy

The Research Portal is Queen's institutional repository that provides access to Queen's research output. Every effort has been made to ensure that content in the Research Portal does not infringe any person's rights, or applicable UK laws. If you discover content in the Research Portal that you believe breaches copyright or violates any law, please contact openaccess@qub.ac.uk. 


\section{Alzheimer's disease-like pathology has transient effects on the brain and blood metabolome}

ARTICLE in NEUROBIOLOGY OF AGING · NOVEMBER 2015

Impact Factor: 5.01 · DOI: 10.1016/j.neurobiolaging.2015.11.014

READS

144

10 AUTHORS, INCLUDING:

\section{Christopher Elliott}

Queen's University Belfast

382 PUBLICATIONS $\quad \mathbf{5 , 5 0 3}$ CITATIONS

SEE PROFILE

Patrick Kehoe

University of Bristol

246 PUBLICATIONS $\quad 7,051$ CITATIONS

SEE PROFILE

\section{Peter Passmore}

Queen's University Belfast

72 PUBLICATIONS 1,023 CITATIONS

SEE PROFILE

Brian D Green

Queen's University Belfast

192 PUBLICATIONS 1,941 CITATIONS

SEE PROFILE 


\section{Alzheimer's disease-like pathology has transient effects on the brain and blood metabolome}

Xiaobei Pan ${ }^{a}$, Muhammad L. Nasaruddin ${ }^{a}$, Christopher T. Elliott ${ }^{a}$, Bernadette McGuinness ${ }^{b}$, Peter Passmore ${ }^{b}$, Patrick G. Kehoe ${ }^{c}$, Christian Hölscher ${ }^{d}$, Paula L McClean ${ }^{e}$, Stewart F. Graham ${ }^{\dagger}$ and Brian D. Green ${ }^{a^{*}}$.

a Advanced Asset Technology Centre, Institute for Global Food Security, Queen's University Belfast, Belfast, UK.

${ }^{b}$ Centre for Public Health, School of Medicine, Dentistry and Biomedical Sciences, Queen's University Belfast, Belfast, UK.

${ }^{c}$ Dementia Research Group, Institute of Clinical Neurosciences, School of Clinical Sciences, University of Bristol, Bristol, UK.

${ }^{d}$ Division of Biomedical and Life Sciences, Lancaster University, Lancaster, UK.

e School of Biomedical Sciences, University of Ulster, Coleraine, UK.

'Beaumont Research Institute, Royal Oak, , USA.

* Corresponding author

Address: Institute for Global Food Security, Queen's University Belfast, N I Technology Centre, 8 Cloreen Park, Northern Ireland, UK.

Tel.: +44(0) 289097 6541; fax: +44 (0) 2890976513

E-mail address: b.green@qub.ac.uk 


\section{Abstract}

The pathogenesis of Alzheimer's disease (AD) is complex involving multiple contributing factors. The extent to which AD pathology impacts upon the metabolome is still not understood, nor is it known how disturbances change as the disease progresses. For the first time we have profiled longitudinally $(6,8,10,12$ and 18 months) both the brain and plasma metabolome of APP/PS1 double transgenic and wild type (WT) mice. A total of 187 metabolites were quantified using a targeted metabolomics methodology. Multivariate statistical analysis produced models that distinguished APP/PS1 from WT mice at 8, 10 and 12 months. Metabolic pathway analysis found perturbed polyamine metabolism in both brain and blood plasma. There were other disturbances in essential amino acids, branched chain amino acids and also in the neurotransmitter serotonin. Pronounced imbalances in phospholipid and acylcarnitine homeostasis was evident in two age groups. AD-like pathology therefore impacts greatly on both the brain and blood metabolomes, although there appears to be a clear temporal sequence whereby changes to brain metabolites precede those in blood.

Keywords: Alzheimer's disease, metabolites, metabolomics, blood, brain, APP/PS1.

\section{Introduction}

Dementia mainly affects the elderly, with the prevalence doubling every five years over the age of (Prince et al., 2014). Alzheimer's disease (AD) is a progressive and fatal neurodegenerative disorder and the most common form of dementia, accounting for $60-80 \%$ of all dementia cases (Prince et al., 2014). AD is clinically characterised by progressive memory loss, mood changes, problems with communication and reasoning, and eventual loss of independent living. Familial AD (FAD), often associated with an earlier-onset ( $<65$ years of age), is an autosomal dominant form of $A D$ caused by mutations in the genes encoding 
amyloid precursor protein (APP) and presenilins 1 and 2 (PS1 and PS2) leading to the subsequent accumulation of $\beta$-amyloid (A $\beta$ ) (Borchelt et al., 1997; Jankowsky et al., 2004; Selkoe, 2001; Selkoe and Schenk, 2003). AD is characterised by the pathological accumulation of extracellular $A \beta$ and abnormally phosphorylated tau filaments in neurons that lead to senile plaques and neurofibrillary tangles (NFTs), respectively (Blennow et al., 2006; Selkoe, 2004; Skovronsky et al., 2006). Transgenic mouse models containing mutations in the human APP and/or PS1 genes are widely used in experimental studies to investigate the pathophysiological role of $A \beta$ in early-onset AD patients. The APPswe/PS1deltaE9 (APP/PS1) strain is one such example that has been extensively characterised and utilised. These mice develop $A \beta$ plaques at 5-6 months of age, although production of $A \beta$ has been shown to occur as early as 3 months in the form of both $A \beta(1-40)$ and (1-42) (Volianskis et al., 2010). APP/PS1 mice display progressive age-related impairments in memory that appear as early as 7 months of age (Volianskis et al., 2010; Xiong et al., 2011). In behavioural tests the mice show deficits in measuring spatial navigation and reference learning (Xiong et al., 2011). Although APP/PS1 mice do not model all facets of human $A D$, they do enable longitudinal investigations not normally possible in people in a clinical environment.

Metabolomics is the scientific investigation of chemical processes involving metabolites. Metabolomics techniques can comprehensively and simultaneously help to measure disturbances in metabolic pathways that reflect changes downstream from genomic, transcriptomic and proteomic systems in a high-throughput manner (Beckonert et al., 2007; Fiehn, 2002). It holds considerable potential as a discovery platform for identifying novel diagnostic biomarkers for AD but also many other neurodegenerative diseases. Metabolomics studies have previously been undertaken in APP/PS1 mice (Chen et al., 2012; GonzalezDominguez et al., 2015a; González-Domínguez et al., 2014; Graham et al., 2013b; Marjanska et al., 2005; Trushina et al., 2012; Yao et al., 2009), however, the majority of these studies (including our own (Graham et al., 2013b)) suffer from limitations commonly befalling many metabolomics investigations conducted to date. The current study was designed having noted 
earlier approaches to undertake a more robust metabolomics evaluation of this important model of AD. Many previous studies had inadequate consideration of the optimal experimental design, a common arguable limitation being the use of arbitrary sample sizes without formal statistical power calculations. Another common limitation was a cross-sectional approach examining a single time point - therefore providing only the narrowest of windows through which to view and obtain reliable biological information. The majority of previous studies were also restricted to one sample type in isolation and did not examine whether biochemical alternations were more widespread. Finally, all potential sources of biological variation (i.e. potential confounders) were not always minimised in the experimental design, such as considering the gender of animals and subjects that can have a strong influence on the metabolome (Dunn et al., 2015; Graham et al., 2013a; Qiao et al., 2011). The current study undertook a targeted and quantitative methodology with optimal sample size pre-calculated to achieve $100 \%$ statistical power. A total of 187 pre-nominated metabolites were measured in both brain and blood samples from female animals and this included amino acids, biogenic amines, phospholipids and acylcarnitines.

Earlier metabolomics studies have revealed a number of biochemical disturbances in APP/PS1 mice. Previous studies using in vivo proton magnetic resonance spectroscopy $\left({ }^{1} \mathrm{H}\right.$ MRS) found decreases in $\mathrm{N}$-acetylasparatate and glutamate, and an increase in myo-inositol concentrations in APP/PS1 mice (Chen et al., 2012; Marjanska et al., 2005). Glycolytic pathways involving the Kreb's cycle, and neurotransmitter and amino acid metabolism, were found to be significantly affected in APP/PS1 mouse brain (Trushina et al., 2012). Furthermore, ${ }^{1} \mathrm{H}$ NMR metabolomics studies found altered ascorbate, creatine, $\mathrm{Y}$-aminobutyric acid and NAA in APP/PS1 mouse brain, and altered acetate, citrate, glutamine and methionine in blood plasma (Graham et al., 2013b). A recent study applying GC-MS and UPLC-MS investigated the metabolic perturbations in five brain regions of APP/PS1 mice at 6 months of age (González-Domínguez et al., 2014). Region-specific alterations were observed for some metabolites associated with abnormal fatty acid composition of phospholipids and 
sphingomyelins (SPHs), or differential regulation of neurotransmitter amino acids (e.g. glutamate, glycine, serine, $\mathrm{N}$-acetyl-aspartate). Disturbances in phospholipids, energy deficiencies, altered homeostasis of amino acid and oxidative stress in APP/PS1 mouse spleen and thymus were also observed (Gonzalez-Dominguez et al., 2015a). One study employing HPLC-ELSD compared the cortical levels of cholesterol and phospholipid subclasses at ages 4 and 9 months (Yao et al., 2009), and found that membrane lipids of APP/PS1 mice including cholesterol and phospholipid were significantly decreased at 9 months (Yao et al., 2009). Among phospholipid subclasses, phosphatidylethanolamine (PE), phosphatidylserine (PS) and phosphatidylcholine (PC) were selectively reduced (Yao et al., 2009). Despite the fact that metabolomics studies have pinpointed some metabolites affected by the development of $A D$-like pathology the findings are often conflicting, fragmented and incongruent. The aim of this study was to longitudinally study the profile of pre-defined metabolites in an important and widely used transgenic AD model over much of its lifespan, and to monitor disturbances close to the initial pathological insult as well as those that arise within the blood circulation.

\section{Material and methods}

\subsection{Brain tissue and plasma from APP/PS1 mouse}

Founder APPswe/PS1DE9 (APP/PS1) male mice were initially obtained from the Jackson lab (USA), and bred at the Ulster University. Heterozygous males were bred with wild-type (WT) C57/BI6 females bought locally (Harlan, UK). APP/PS1and WT mice were housed under identical conditions and fed the same rodent maintenance diet (14\% fat, $32 \%$ protein, and $54 \%$ carbohydrate; total energy of $3.0 \mathrm{kcal} / \mathrm{g}$; Harlan).

APP/PS1 mice are a transgenic C57BL/6J mouse model co-expressing the Swedish mutation (K595N/M596L) and the deltaE9 PS-1 exon deletion (mutated human presenilin-1) (Lalonde et al., 2005). Offspring were tail snipped and genotyped using PCR. PCR used 
primers specific for the APP sequence (Forward "GAATTCCGACATGACTCAGG", Reverse: "GTTCTGCTGCATCTTGGACA"). Mice not expressing the transgene were used as WT controls. For this study, female APP/PS1dE9 mice, aged 6, 8, 10, 12 and 18 months and, age matched WT female C57BL/6littermate controls $(n=8-9)$ were used. Mice were fasted for 16 h, deeply anaesthetised with pentobarbitol, and blood samples were collected into heparinised tubes, centrifuged for 30 seconds at $13,000 \times g$ and the resulting plasma were stored at $-80^{\circ} \mathrm{C}$ prior to metabolomics investigations. Whole mouse brain was also collected and snap-frozen in liquid nitrogen and stored at $-80^{\circ} \mathrm{C}$ until further use.

\subsection{Brain tissue extraction}

Mouse brain samples were collected into individual tubes to avoid cross-contamination, then lyophilized and cryogenically milled to a fine dry powder. Powdered PM brain tissue (25 $\mathrm{mg} \pm 0.5 \mathrm{mg}$ ) was extracted in $300 \mu \mathrm{L}$ in a solvent ( $85 \%$ ethanol and $15 \%$ PBS buffer) previously optimised for brain metabolite profiling (Urban et al. 2010). The samples were sonicated (5 min), vortexed (30 sec), centrifuged at $\left(10,000 \mathrm{~g} ; 4^{\circ} \mathrm{C} ; 5 \mathrm{~min}\right)$ and the supernatant retained for analysis.

\subsection{Targeted metabolomics}

Quantitative mass spectrometry-based metabolomic profiling was performed using the Biocrates AbsoluteIDQ p180 (BIOCRATES, Life Science AG, Innsbruck, Austria), as previously described (Nkuipou-Kenfack et al., 2014; Roemisch-Margl et al., 2012). The AbsolutelDQ p180 kit provides simultaneous quantification of amino acids, acylcarnitines, SPHs, PCs, hexose (glucose), and biogenic amines in many biological samples. The samples were processed according to the manufacturer's instructions and analysed on a triplequadrupole mass spectrometer (Xevo TQ-MS, Waters Corporation, Milford, USA). The data were recorded in a 96-well format and seven calibration standards were integrated in the kit. 
Human EDTA plasma samples spiked with standard metabolites were used as quality control samples to assess reproducibility of the assay. Briefly, $10 \mu \mathrm{L}$ of mouse plasma samples and $10 \mu \mathrm{L}$ of $\mathrm{PM}$ brain extract (prepared as described above) were used for the targeted metabolomics analysis. The amino acids and biogenic amines were derivatised using phenylisothiocyanate (PITC) in the presence of isotopically labelled internal standards internal standards as detailed by the manufacturer. This was followed by separation using a UPLC (IClass, Waters Corporation, Milford, USA) and quantified using a triple-quadrupole mass spectrometer (Xevo TQ-MS, Waters Corporation, Milford USA) operating in the multiple reaction monitoring (MRM) mode. All the remaining metabolites were quantified using the same mass spectrometer without column separation by the flow injection analysis (FIA) operating in MRM mode. Metabolite concentrations were calculated and expressed as $\mu \mathrm{mol} / \mathrm{mg}$ tissue.

\subsection{Statistical analysis}

Sample size and power were estimated based on data (i.e. mean concentrations and standard deviations) obtained during previous laboratory sampling of human plasma using the Biocrates AbsoluteIDQ p180 kit. An on-line tool "Inference for Means: Comparing Two Independent Samples" developed by the University of British Columbia, Department of Statistics was used to calculate statistical power with the type I error rate set at the default value of 0.05 [http://www.stat.ubc.ca/ rollin/stats/ssize/n2.html]. It was calculated that for most metabolite measurements a samples size of $n \geq 8$ gave desired power of $1.0(100 \%)$. The concentrations of 187 metabolites were compared exported to Simca 13 (Umetrics, Umea, Sweden) for multivariate analysis. Data were log transformed, pareto-scaled and grouped into APP/PS1 and WT prior to analysis using orthogonal projection to latent structures-discriminant analysis (OPLS-DA). Data were tested for normality and found to be non-normally distributed (SPPS version, 13.0). Non-parametric one-way analysis of variance analysis (Kruskal-Wallis) was performed to determine if metabolites were statistically different across the 10 groups 
$(p<0.05)$. Metabolic pathway analysis was performed using online metabolomics tools (Metaboanalyst (Xia et al., 2012) and Vanted (version 2.0.1) (Junker et al., 2006)). Heat map visualisations of data were created using PermutMatrix version 1.9.3.0 (Caraux and Pinloche, 2005).

\section{Results}

\subsection{The effect of age on the brain and plasma metabolome of APP/PS1 mice.}

Initial assessments were made to ascertain the overall extent of changes in brain and plasma metabolites. Multiple comparison testing provided an overview of how metabolites in each metabolite class were affected at each age time point and in each sample type, which is detailed in Table 1. Multivariate analysis was used to build models differentiating all 10 groups analysed for brain (Figure 1A) and plasma (Figure 1B). Supervised orthogonal projection to least squares discriminant analysis (OPLS-DA) was employed to visually discriminate between WT (circles) and APP/PS1 (triangles) for mice aged 6 months (blue), 8 months (red), 10 months (green), 12 months (yellow) and 18 months (grey). The scores plots (Figure 1) showed that was possible to visibly discern WT from APP/PS1 mice at all ages with the exception of 6 and 18 months were for both brain and plasma there was some degree of overlap. This observation was confirmed when OPLS-DA was applied at each time point to assess how accurately class membership was predicted (Q2 cumulative; Table 1). The predictive power of the models (Q2) varied according to age in both brain and plasma but some of the lowest Q2 values occurred at 6 and 18 months. Conversely, models with the greatest predictive ability were at 8 months $(Q 2=0.836)$ and 12 months $(Q 2=0.890)$, for brain and plasma, respectively.

\subsection{Metabolic Pathway Analysis of separate tissue compartments of APP/PS1 mice.}


Multiple comparison testing demonstrated that there were significant concentration changes in a number of amino acids and polyamines in both blood and brain samples. Metabolic pathway analysis made it possible to visualise the biochemical relationships of these metabolites and the ages at which they were affected (Figures 2 and 3). Significant increases in the polyamines putrescine, spermidine and spermine $(p<0.05)$ in APP/PS1 mice were detected in both brain and plasma. Disturbances of these metabolites occurred earlier (6-8 months) in brain than in plasma (10 months). Concentrations of plasma glutamine $(p<0.05)$ and citrulline $(p<0.01)$ were lower in APP/PS1 mice at 12 but neither of these precursor metabolites were affected in brain. Threonine levels were lower in APP/PS1 brain tissue (10 months; $p<0.05)$ and in plasma (12 months; $p<0.01)$. Serine was also significantly altered (10 months; $p<0.05)$ in both the brain and plasma of APP/PS1 mice. Contrastingly, arginine was only significantly altered $(6$ months; $p<0.05)$ in brain tissue, whilst concentrations of glutamine and proline were recorded as being significantly different in plasma $(p<0.05)$.

\subsection{Disturbances in amino acid levels between APP/PS1 and WT mice}

Numerous other changes in amino acid and biogenic amines unrelated to the polyamine pathway also were also identified (Table 2). However, only two amino acids (phenylalanine and tyrosine) were affected in both brain and blood, and only phenylalanine showed the same direction of change (i.e. increased) in both sample types. Although, phenylalanine levels changed first in brain ( 6 months) and then in plasma (10 months).

Many more disturbances in metabolite concentrations were observed in plasma than brain tissue. Interestingly five essential amino acids (EAAs), histidine, phenylalanine, valine, isoleucine, methionine were affected as were two of the three branched-chain amino acids (BCAAs). Following this we assessed the overall changes in BCAAs and the ratio of essential amino acids to non-essential amino acids (EAAs:NEAAs; Supplementary Table 1). In APP/PS1 plasma the EAAs:NEAAs ratio was significantly lower at 6 months but was 
significantly higher at 12 and 18 months. The total level of BCAAs was unaffected in brain and plasma of all age groups.

Strikingly, at 10 months APP/PS1 plasma displayed a number of marked increases in amino acid levels. This included histidine and phenylalanine (increased 16-18\%) but also methionine and methionine sulfoxide which were increased $36 \%$ and $73 \%$ respectively. Furthermore, asparagine and taurine were increased $37 \%$ and $28 \%$, respectively. Serotonin was significantly increased in the plasma taken from APP/PS1 mice at 8 months (72.7\%). At 12 months a-aminoadipic acid was $68 \%(p<0.001)$ lower in APP/PS1 mice compared to WT controls.

\subsection{Phospholipid alterations}

The alteration of 105 membrane lipid metabolites including 17 lysophosphatidylcholines (LysoPCs), 73 PCs and 15 SPHs were assessed for both plasma and brain tissue samples. Changes in both physiological compartments were visualised using heat maps (Figure 5). Metabolites in red were higher in APP/PS1 compared to WT controls and those in green were lower. One PC (PC aa C36:0) was not measureable in brain and 5 SPHs (SM (OH) C22:1, SM $(\mathrm{OH}) \mathrm{C} 24: 1, \mathrm{SM}$ C20:2, SM C26:0 and SM C26:1) were not measurable either in brain tissue or in plasma. The heat map revealed statistically significant and widespread increases in PC levels in APP/PS1 brain tissue at 8 months of age. Also evident was the widespread and statistically significant decrease of phospholipid levels in APP/PS1 plasma at 12 months. From 72 PCs measured in brain tissue, 28 were significantly higher $(p<0.05)$ in APP/PS1 mice at 8 months. Of the 73 PCs measured in plasma, 51 were significantly lower $(p<0.05)$ in APP/PS1 mice at 12 months. Of the 17 LysoPCs, 2 LysoPCs (LysoPC a C16:1 and LysoPC a C28:0) were significantly higher in APP/PS1 brain at 8 months $(p<0.05)$. A total of 7 LysoPCs (LysoPC a C14:0, LysoPC a C16:0, LysoPC a C16:1, LysoPC a C17:0, LysoPC a C18:1, LysoPC a C18:2 and LysoPC a C20:4) were significantly lower in APP/PS1 plasma $(p<0.05)$ at 12 months. Furthermore, of the 10 measured SPHs, 6 SPHs (SM (OH) C16:1, SM C16:0, 
SM C18:0, SM (OH) C22:2, SM C24:0 and SM C24:1) were at significantly higher concentrations in APP/PS1 brain at 8 months and 3 SPHs (SM $(\mathrm{OH}) \mathrm{C} 14: 1, \mathrm{SM}(\mathrm{OH}) \mathrm{C} 22: 1$ and SM C24:0) were at significantly lower concentrations in APP/PS1 plasma $(p<0.05)$ at 12 months. Changes in the total levels of each phospholipid class were also examined (Supplementary Table 2). In APP/PS1 brain the total levels of PC and SPH were significantly higher at 8 months. Contrastingly, in APP/PS1 plasma the total levels of PC and LysoPC were significantly lower at 12 months.

\subsection{Acylcarnitine alterations}

Of the 40 acylcarnitines quantified 8 significant differences in brain tissue and 8 were significantly different in plasma $(p<0.05)$ (Figure 4). Only 2 acylcarnitines were significantly altered in both brain and plasma $(\mathrm{C} 10: 1, \mathrm{C} 12: 1)$ and neither of these $(\mathrm{C} 10: 2)$ exhibited the same direction of change.

In APP/PS1 plasma the most marked acylcarnitine changes were the $65-96 \%$ reductions $(p<0.01)$ in the levels of $C 4: 1$ and $C 6: 1$ which persisted from the ages of 8 months onwards. In APP/PS1 brain the most marked changes were the $45-68 \%$ increases $(p<0.05)$ in the levels of C7-DC, C10:1 and C12:1 occurring at 8, 10 and 18 (but not 12) months.

\section{Discussion}

This is the first high-throughput targeted metabolomics analysis to longitudinally investigate the metabolic disturbances of APP/PS1 mice. The aim was to identify the specific metabolite changes consequential to the development of AD-like pathology in these mice, and how these changes are affected as pathology progresses over time. The study also assessed which neurometabolite changes are mirrored within the blood circulation as a method to explore what, if any, peripheral markers may provide insights into brain metabolite behaviour.

A total of 187 metabolites were quantified including amino acids, biogenic amines, phospholipids and acylcarnitines. One of the key findings is that the majority of metabolite perturbations in the APP/PS1 model are transient. The majority of perturbations do not persist, 
as might have been expected in chronic and progressive disease pathology. Our findings suggest that there are key periods when wide-ranging metabolic disturbances occur, and the data contained herein could be highly informative, both for future experimentation with this animal model, but also in terms of interpreting metabolomics findings from human samples.

The targeted metabolomics approach undertaken was able to discern APP/PS1 mice from WT most effectively at ages between 8 and 12 months. However, for the both the youngest ( 6 months) and the eldest (18 months) age groups it was much less clear as there was some degree of overlap between WT and APP/PS1. This was the case for both plasma and brain models. It was possible to quantify the specificity of OPLS-DA models for each age group. It was evident that predictive scores (Q2) were poorer at 6 months when $A \beta$ plaque formation is in the early stages and memory impairment is minimal (Hamilton and Holscher, 2012; Volianskis et al., 2010). However, it is very interesting that predictive scores were also quite poor at 18 months when there is extensive plaque formation and neurogenesis is almost completely suppressed (Hamilton and Holscher, 2012). This suggests either that the APP/PS1 model eventually returns to a more balanced metabolic state, or that the aging of WT mice leads to a metabolite profile more akin to the APP/PS1. It is also worth noting that some multivariate models were particularly impressive. For example, plasma metabolites at 12 months predicted APP/PS1 mice with $89 \%$ accuracy, and brain metabolites at 8 months predicted with $84 \%$ accuracy. This targeted approach is a significant improvement on the predictive abilities of our earlier NMR-based profiling approaches that predicted APP/PS1 mice (12 months) with $62 \%$ and $74 \%$ for brain and plasma samples, respectively (Graham et al., 2013b).

Taking into consideration the overall changes in the metabolite classes (as outlined in Table 1) it is unsurprising that changes in amino acids/biogenic amines and phospholipids where fundamentally important in distinguishing APP/PS1 from WT. For phospholipids, these were most significantly disturbed at 8 months in brain and 12 months in plasma. For amino 
acids/biogenic amines these were affected to the greatest extent at 10 months in both brain and plasma.

Polyamine metabolism was transiently disturbed in both brain and plasma. Increased levels of three polyamines, putrescine, spermidine and spermine, have been reported in brain tissue and plasma from AD patients (Inoue et al., 2013; Trushina et al., 2013). The underlying explanation for this is that $A \beta$ causes up-regulation of polyamine uptake and increased ornithine decarboxylase activity, which leads to increased polyamine synthesis (Yatin et al., 1999; Yatin et al., 2001). Polyamines, like spermidine and spermine are positive modulators of the N-Methyl-D-Aspartate (NMDA) receptor. Thus, increased polyamine synthesis can cause over activity of the NMDA receptor that in turn leads to neuronal excitotoxicity that is very common in AD (Cacabelos et al., 1999). Indeed, it is notable that the most recently licensed treatment for $A D$ is the NMDA receptor antagonist memantine and the current findings that brain polyamine elevations occur early in the pathology of the mice studied is consistent with this. Putrescine that precedes both spermidine and spermine in the biochemical pathway is the first to be elevated (6 months) in APP/PS1 mice. This elevation of putrescine could stem from the observed rise in its precursor metabolite, arginine. The subsequent increase in both brain spermidine and spermine occurred at 8 months. These observations were mirrored in blood plasma where there was a very similar trend, albeit later, with plasma elevations of putrescine, spermidine and spermine occurring at 10 months. It remains to be proven whether the polyamine disturbances we detected in the circulation are the direct result of the observed cerebral disturbances. It is also possible that these arise spontaneously and independently in peripheral systems of the APP/PS1 mouse. This still has to be determined, however, putrescine is deceased in the thymus of APP/PS1 mice and is entirely absent from the spleen, which eliminates these organs as potential sources for elevated blood polyamine levels (Gonzalez-Dominguez et al., 2015a). It is interesting to compare these findings with those from human subjects. We recently reported an untargeted metabolomics analysis of human plasma whereby people with Mild Cognitive Impairment (MCl) 
that remain stable over time ( $\mathrm{MCl}$-stable) and people with $\mathrm{MCl}$ who later converted to $\mathrm{AD}$ ( $\mathrm{MCl}$-converters) demonstrated significant increases in arginine compared with a cognitively normal age-matched control group (Graham et al., 2015). Furthermore, the fate of this arginine differed substantially between these groups. In the $\mathrm{MCl}$-converters group putrescine, as with the mice studied here, was channelled towards the production of spermidine and spermine. In contrast $\mathrm{MCl}$-stable group had their putrescine diverted towards the production of $\mathrm{N}$ acetylputrescine or 4-aminobutanal (the latter were not measured in the present study). Thus the findings presented here strengthen the suitability of our mouse model as they recapitulate similar behaviour for the elevated putrescine, spermidine and spermine in human plasma. Furthermore, it does appear that there is some selectivity towards the polyamine pathway in $A D$, since other metabolites downstream of arginine (e.g. creatinine, sarcosine, ornithine, proline or hydroxyproline) are either unaffected or very inconsistently affected. These data support the need for further investigation of polyamine metabolism in AD research, particularly since chemical inhibition of the polyamine system counteracts $A \beta$ induced memory impairments, reportedly through modulation of extra-synaptic NMDA receptor signalling (Gomes et al., 2014).

Beyond these biochemical pathways, numerous other metabolite disturbances were evident in the APP/PS1 mouse model. First, we observed a number changes in the plasma levels of amino acids. We observed five EAAs which were altered only in plasma and this included two of the three BCAAs. The magnitude of these amino acid disturbances was sufficiently large enough to impact on EAAs:NEAAs but not the overall levels of BCAAs. It is possible that the period of fasting (undertaken to stabilise key physiological biomarkers) could favour changes in amino acids which may arise from accelerated loss of liver function in AD. It is also possible that EEAs and BCAAs changes are a consequence of the neuronal destruction or degenerative brain pathology, which occurs in this model (Hamilton \& Holscher 2012). 
We also found a number of other important metabolites that were altered in APP/PS1 plasma including the neurotransmitter serotonin, which was increased by $>70 \%$ at 8 months. It is not entirely clear why there was a significant decrease in $\alpha$-aminoadipic acid in APP/PS1 mice, but since this is an intermediates in amino acid metabolism it could be a consequence of the wider amino acid disturbances. As far as we are aware, changes in a-aminoadipic acid have not been reported before but the potential relevance that it is a NMDA receptor ligand. In fact the L-enantiomeric form exerts an agonist activity, while the D-enantiomeric form acts as an antagonist (Brauner-Osborne et al., 2000; Guldbrandt et al., 2002). Since NMDA has been implicated in both cell death and neuronal excitotoxicity in AD (Butterfield and Pocernich, 2003), clearer delineation of the relative abundance of the D-and L-enantiomers of $\alpha$ aminoadipic acid, would be useful to allow better interpretation of how these might modulate neurotoxicity via NMDA receptors with respect to AD pathology. At 10 months both methionine and methionine sulfoxide were significantly elevated in APP/PS1 mice (36 and 73\%, respectively). This is supported by a study that found modest non-significant increases in methionine in serum from people with $\mathrm{MCl}$ but significantly reduced levels in $A D$ patients (Gonzalez-Dominguez et al., 2014). Also noteworthy is that the levels of methionine sulfoxide reductase (Msr) enzymes are elevated by $40 \%$ in APP/PS1 mice at 9 months of age (Moskovitz et al., 2011), which may suggest that upregulation of Msr in the early stages of AD may be an attempt to mitigate oxidative stress, ultimately failing in the later stages of the disease.

Detailed glycerophospholipid and sphingolipid lipid profiling of the APP/PS1 mouse was also undertaken in this study. Glycerophospholipids (PCs and LysoPCs) are the major class of complex lipids playing essential roles in neural membrane formation and intraneuronal signal transduction (Farooqui et al., 2000; Farooqui et al., 2007). PCs are the most abundant glycerophospholipids that have a choline polar headgroup attached to the phosphate group. The LysoPCs result from partial hydrolysis of PCs, which removes one of the fatty acid groups. This hydrolysis is generally thought to be the result of the enzymatic action of phospholipase 
A2 (PLA2) (Farooqui et al., 2000). Our results demonstrate that there are 2 time periods when PCs undergo considerable and widespread flux. First, in the APP/PS1 brain at 8 months almost 40\% PCs (28 from 72) increased significantly. Second and in contrast, in APP/PS1 plasma an even higher proportion (70\%) of PCs (51 from 73 analysed) significantly decreased. It well known that PCs are the most abundant phospholipid in the brain, and it is also known that uptake of choline from the circulation into the brain decreases with advancing age. It has been suggested that reduced uptake of plasma choline could lead to increased degradation of membrane PC in order to produce sufficient amounts of the neurotransmitter acetylcholine (Mi et al. 2013).

Such detailed phospholipid profiling has been undertaken is this model before, however, the aggregate levels of PCs in APP/PS1 mouse brain have been reported to be lower at 9 months of age (Yao et al., 2009). It has also been noted that total phosphatidylcholine diacyl (PCaa) levels tend to be lower in APPswe brain (Grimm et al., 2011). However our findings are in broad agreement with a previous study that demonstrated the APP/PS1 mouse brain exhibits increased SPHs and PCs at 9 months (Fabelo et al., 2012). The potential relevance of such a finding is that neural membrane PCs are hydrolysed preferentially by the enzyme $\mathrm{PLA}_{2}$ to generate LysoPCs and free fatty acids (Larsson Forsell et al., 1999; Mancuso et al., 2000; Prokazova et al., 1998), but the importance of $P L A_{2}$ in AD pathogenesis is contentious (Gattaz et al., 1996; Gattaz et al., 1995; Stephenson et al., 1996). Taken together, the data from these studies suggests that $\mathrm{PLA}_{2}$ activity (particularly $\mathrm{cPLA}$ and $\mathrm{PLA} \mathrm{A}_{2}$ ) is reduced, resulting in reduced phospholipid turnover in early $A D$ pathogenesis. This could contribute to cognitive dysfunction and neuropathology in the early stages of the disease. As the disease worsens, $\mathrm{CPLA}_{2}$ and SPLA 2 levels (and accordingly the metabolism of phospholipids) become elevated in AD brains (Schaeffer et al., 2009; Schaeffer and Gattaz, 2008). This may explain why we observed a general increase in PCs in the brain at 8 months and why subsequently this increase is not sustained. 
More than one third of all the SPHs measured in APP/PS1 mouse brain were significantly elevated at 8 months of age $(p<0.05)$. SPHs are precursors for ceramide production. Their accumulation induces apoptosis and seems to worsen neurodegeneration by increasing $A \beta$ biosynthesis and promoting gamma-secretase processing of APP (Cutler et al., 2004; Grimm et al., 2005; Puglielli et al., 2003). Our findings closely resemble other work showing that global brain levels of ceremides, sphingolipids and related molecules are increased in the cerebral cortex of APPSL/PS1Ki and APP/PS1 mice especially after the age of 6 months (Barrier et al., 2010). Interestingly, the concentrations of SPH were nearly constant between 3 \& 6 months old APPSL/PS1Ki mice (Barrier et al., 2010). We also found a number of changes in acylcarnitine species in both brain and plasma from APP/PS1 mice. We could not detect any particular overall pattern in acylcarnitine changes. However, as a class acylcarnitines were rather unique because some metabolites were very persistently affected. Most conspicuously C4:1 and C6:1 were decreased $>65 \%$ in APP/PS1 plasma at 8, 10, 12 and 18 months.

This study has identified a series of changes in a number of metabolic networks including those involved in membrane lipid metabolism and neuronal health and neurotoxicity, as well as altered amino acid metabolism. It is important to remember that anaesthesia will have affected the physiological status of mice and could have indirectly influenced the levels some metabolites measured here. However, there are consistencies with the findings of another recent study of plasma taken from 6 month old APP/PS1 mice although there are some differences in the lead metabolites found in this study (Gonzalez-Dominguez et al., 2015b). What is important in our study, and which contrasts with the recent study is that we have delineated some of the key metabolomics impairments occurring longitudinally over the lifespan of the APP/PS1 mouse $(6,8,10,12$ and 18 months). Our longitudinal approach has provided an insight into the different temporal phases of metabolic disturbances that may occur both centrally and peripherally in human $A D$ as a result of $A \beta$ pathology. There appear to be certain periods in which this mouse model underwent widespread metabolic flux, initially occurring in the brain followed 2 to 4 months later with related changes in circulating 
metabolites. We found these disturbances were frequently transient in nature neither being progressively nor persistently affected. These were only evident from our broader timeframe of longitudinal assessment compared to other studies, and occurred in mice at ages that were older than those recently reported (Gonzalez-Dominguez et al., 2015b).

\section{Conclusion}

In conclusion, this study provides strong evidence to support the value of investigating metabolic changes in mice modelling certain aspects of AD pathology. These provide a basis to refine studies for future translation in humans such as further examination of changes in PC species that we observed both in the central nervous system and circulation and that may provide a means to tracking the pathophysiology of AD. In addition to supporting other studies of the involvement of certain metabolic pathways that appear to be affected in relation to models of $A \beta$ pathology, in this study, the depiction of how polyamine metabolism is disturbed is potentially relevant towards future therapies based on the development of inhibitors of the polyamine system.

\section{Acknowledgement}

This work is supported by grants from Alzheimer's Research UK [ARUK-NCH2012B-5; grant ARUK-PPG2011B-8, ARUK-Network2012-11 and ARUK-Network2014-16], and a by Proof of Concept grant from Invest Northern Ireland [INI-PoC406]. We also gratefully acknowledge assistance from the European Regional Development Fund (ERDF) supporting the Advanced ASSET Centre.

\section{Disclosure statement}

The authors have no conflicts of interest to disclose. 


\section{References}

Barrier L, Fauconneau B, Noel A, Ingrand S. Ceramide and related sphingolipid levels are not altered in disease associated brain regions of APP and APP/PS1 mouse models of Alzheimer's disease: relationship with the lack of neurodegeneration? Int J Alzheimers Dis $2010 ; 2011$.

Beckonert O, Keun HC, Ebbels TMD, Bundy JG, Holmes E, Lindon JC, Nicholson JK. Metabolic profiling, metabolomic and metabonomic procedures for NMR spectroscopy of urine, plasma, serum and tissue extracts. Nat Protoc 2007;2:2692-2703.

Blennow K, de Leon MJ, Zetterberg H. Alzheimer's disease. Lancet 2006;368:387-403.

Borchelt DR, Ratovitski T, vanLare J, Lee MK, Gonzales V, Jenkins NA, Copeland NG, Price DL, Sisodia SS. Accelerated amyloid deposition in the brains of transgenic mice coexpressing mutant presenilin 1 and amyloid precursor proteins. Neuron 1997;19:939-945.

Brauner-Osborne H, Egebjerg J, Nielsen EO, Madsen U, Krogsgaard-Larsen P. Ligands for glutamate receptors: Design and therapeutic prospects. J Med Chem 2000;43:26092645.

Butterfield DA, Pocernich CB. The glutamatergic system and Alzheimer's disease Therapeutic implications. Cns Drugs 2003;17:641-652.

Cacabelos R, Takeda M, Winblad B. The glutamatergic system and neurodegeneration in dementia: Preventive strategies in Alzheimer's disease. Int $\mathrm{J}$ Geriatr Psychiatry 1999;14:3-47.

Caraux G, Pinloche S. PermutMatrix: a graphical environment to arrange gene expression profiles in optimal linear order. Bioinformatics 2005;21:1280-1281.

Chen S-q, Cai Q, Shen Y-y, Wang P-j, Teng G-j, Zhang W, Zang F-c. Age-related changes in brain metabolites and cognitive function in APP/PS1 transgenic mice. Behav Brain Res 2012;235:1-6.

Cutler RG, Kelly J, Storie K, Pedersen WA, Tammara A, Hatanpaa K, Troncoso JC, Mattson MP. Involvement of oxidative stress-induced abnormalities in ceramide and cholesterol 
metabolism in brain aging and Alzheimer's disease. Proc Natl Acad Sci U S A 2004;101:2070-2075.

Dunn WB, Lin W, Broadhurst D, Begley P, Brown M, Zelena E, Vaughan AA, Halsall A, Harding N, Knowles JD, Francis-Mclntyre S, Tseng A, Ellis DI, O'Hagan S, Aarons G, Benjamin B, Chew-Graham S, Moseley C, Potter P, Winder CL, Potts C, Thornton P, McWhirter C, Zubair M, Pan M, Burns A, Cruickshank JK, Jayson GC, Purandare N, Wu FC, Finn JD, Haselden JN, Nicholls AW, Wilson ID, Goodacre R, Kell DB. Molecular phenotyping of a UK population: defining the human serum metabolome. Metabolomics 2015;11:9-26.

Fabelo N, Martin V, Marin R, Santpere G, Aso E, Ferrer I, Diaz M. Evidence for premature lipid raft aging in APP/PS1 double transgenic mice, a model of Familial Alzheimer Disease. J Neuropathol Exp Neurol 2012;71:868-881.

Farooqui AA, Horrocks LA, Farooqui T. Glycerophospholipids in brain: their metabolism, incorporation into membranes, functions, and involvement in neurological disorders. Chem Phys Lipids 2000;106:1-29.

Farooqui AA, Horrocks LA, Farooqui T. Interactions between neural membrane glycerophospholipid and sphingolipid mediators: A recipe for neural cell survival or suicide. J Neurosci Res 2007;85:1834-1850.

Fiehn O. Metabolomics - the link between genotypes and phenotypes. Plant Mol Biol 2002;48:155-171.

Gattaz WF, Cairns NJ, Levy R, Forstl H, Braus DF, Maras A. Decreased phospholipase A(2) activity in the brain and in platelets of patients with Alzheimer's disease. Eur Arch Psychiatry Clin Neurosci 1996;246:129-131.

Gattaz WF, Maras A, Cairns NJ, Levy R, Forstl H. Decreased phospholipase A(2) activity in Alzheimer brains. Biol Psychiatry 1995;37:13-17.

Gomes GM, Dalmolin GD, Baer J, Karpova A, Mello CF, Kreutz MR, Rubin MA. Inhibition of the polyamine system counteracts beta-amyloid peptide-induced memory impairment in mice: Involvement of extrasynaptic NMDA receptors. PLoS One 2014;9. 
Gonzalez-Dominguez R, Garcia-Barrera T, Vitorica J, Gomez-Ariza JL. Metabolomics reveals significant impairments in the immune system of the APP/PS1 transgenic mice of Alzheimer's disease. Electrophoresis 2015a;36:577-587.

González-Domínguez R, García-Barrera T, Vitorica J, Gómez-Ariza JL. Region-specific metabolic alterations in the brain of the APP/PS1 transgenic mice of Alzheimer's disease. Biochimica et Biophysica Acta (BBA) - Molecular Basis of Disease 2014;1842:2395-2402.

Gonzalez-Dominguez R, Garcia-Barrera T, Vitorica J, Luis Gomez-Ariza J. Deciphering metabolic abnormalities associated with Alzheimer's disease in the APP/PS1 mouse model using integrated metabolomic approaches. Biochimie 2015b;110C:119-128.

Gonzalez-Dominguez R, Garcia A, Garcia-Barrera T, Barbas C, Luis Gomez-Ariza J. Metabolomic profiling of serum in the progression of Alzheimer's disease by capillary electrophoresis-mass spectrometry. Electrophoresis 2014;35:3321-3330.

Graham SF, Chevallier OP, Elliott CT, Holscher C, Johnston J, McGuinness B, Kehoe PG, Passmore AP, Green BD. Untargeted metabolomic analysis of human plasma indicates differentially affected polyamine and L-arginine metabolism in mild cognitive impairment subjects converting to Alzheimer's disease. PLoS One 2015;10:e0119452e0119452.

Graham SF, Chevallier OP, Roberts D, Hoelscher C, Elliott CT, Green BD. Investigation of the Human Brain Metabolome to Identify Potential Markers for Early Diagnosis and Therapeutic Targets of Alzheimer's Disease. Anal Chem 2013a;85:1803-1811.

Graham SF, Holscher C, McClean P, Elliott CT, Green BD. H-1 NMR metabolomics investigation of an Alzheimer's disease (AD) mouse model pinpoints important biochemical disturbances in brain and plasma. Metabolomics 2013b;9:974-983.

Grimm MOW, Grimm HS, Patzold AJ, Zinser EG, Halonen R, Duering M, Tschape JA, De Strooper B, Muller U, Shen J, Hartmann T. Regulation of cholesterol and sphingomyelin metabolism by amyloid-beta and presenilin. Nat Cell Biol 2005;7:11181123. 
Grimm MOW, Groesgen S, Riemenschneider M, Tanila H, Grimm HS, Hartmann T. From brain to food: Analysis of phosphatidylcholins, lyso-phosphatidylcholins and phosphatidylcholin plasmalogens derivates in Alzheimer's disease human post mortem brains and mice model via mass spectrometry. J Chromatogr A $2011 ; 1218: 7713-7722$

Guldbrandt M, Johansen TN, Frydenvang K, Brauner-Osborne H, Stensbol TB, Nielsen B, Karla R, Santi F, Krogsgaard-Larsen P, Madsen U. Glutamate receptor ligands: Synthesis, stereochemistry, and enantiopharmacology of methylated 2-aminoadipic acid analogs. Chirality 2002;14:351-363.

Hamilton A, Holscher $\mathrm{C}$. The effect of ageing on neurogenesis and oxidative stress in the APP(swe)/PS1(deltaE9) mouse model of Alzheimer's disease. Brain Res 2012;1449:83-93.

Inoue K, Tsutsui H, Akatsu H, Hashizume Y, Matsukawa N, Yamamoto T, Toyo'oka T. Metabolic profiling of Alzheimer's disease brains. Science Reprots 2013;3.

Jankowsky JL, Fadale DJ, Anderson J, Xu GM, Gonzales V, Jenkins NA, Copeland NG, Lee MK, Younkin LH, Wagner SL, Younkin SG, Borchelt DR. Mutant presenilins specifically elevate the levels of the 42 residue beta-amyloid peptide in vivo: evidence for augmentation of a 42-specific gamma secretase. Hum Mol Genet 2004;13:159-170.

Junker BH, Klukas C, Schreiber F. VANTED: A system for advanced data analysis and visualization in the context of biological networks. BMC Bioinformatics 2006;7.

Lalonde R, Kim HD, Maxwell JA, Fukuchi K. Exploratory activity and spatial learning in 12month-old APP(695)SWE/co+PS1/Delta E9 mice with amyloid plaques. Neurosci Lett 2005;390:87-92.

Larsson Forsell PK, Kennedy BP, Claesson HE. The human calcium-independent phospholipase A2 gene multiple enzymes with distinct properties from a single gene. European journal of biochemistry / FEBS 1999;262:575-585. 
Mancuso DJ, Jenkins CM, Gross RW. The genomic organization, complete mRNA sequence, cloning, and expression of a novel human intracellular membrane-associated calciumindependent phospholipase A(2). J Biol Chem 2000;275:9937-9945.

Marjanska M, Curran GL, Wengenack TM, Henry PG, Bliss RL, Poduslo JF, Jack CR, Ugurbil K, Garwood M. Monitoring disease progression in transgenic mouse models of Alzheimer's disease with proton magnetic resonance spectroscopy. Proc Natl Acad Sci U S A 2005;102:11906-11910.

Mi W, van Wijk N, Cansev M, Sijben JW, Kamphuis PJ. Nutritional approaches in the risk reduction and management of Alzheimer's disease. Nutrition. 2013;29:1080-1089.

Moskovitz J, Maiti P, Lopes DHJ, Oien DB, Attar A, Liu T, Mittal S, Hayes J, Bitan G. Induction of methionine sulfoxide reductases protects neurons from amyloid beta protein insults in vitro and in vivo. Biochemistry 2011;50:10687-10697.

Nkuipou-Kenfack E, Duranton F, Gayrard N, Argiles A, Lundin U, Weinberger KM, Dakna M, Delles C, Mullen W, Husi H, Klein J, Koeck T, Zuerbig P, Mischak H. Assessment of metabolomic and proteomic biomarkers in detection and prognosis of progression of renal function in chronic kidney disease. PLoS One 2014;9.

Prince M, Albanese E, Guerchet M, Prina M, Prince M, Albanese E, Guerchet M, Prina M: World Alzheimer Report 2014. London, Alzheimer's Disease International, 2014.

Prokazova NV, Zvezdina ND, Korotaeva AA. Effect of lysophosphatidylcholine on transmembrane signal transduction. Biochemistry-Moscow 1998;63:31-37.

Puglielli L, Ellis BC, Saunders AJ, Kovacs DM. Ceramide stabilizes beta-site amyloid precursor protein-cleaving enzyme 1 and promotes amyloid beta-peptide biogenesis. J Biol Chem 2003;278:19777-19783.

Qiao Q, Li T, Sun J, Liu X, Ren J, Fei J. Metabolomic analysis of normal (C57BL/6J, 129S1/SvImJ) mice by gas chromatography-mass spectrometry: Detection of strain and gender differences. Talanta 2011;85:718-724. 
Roemisch-Margl W, Prehn C, Bogumil R, Roehring C, Suhre K, Adamski J. Procedure for tissue sample preparation and metabolite extraction for high-throughput targeted metabolomics. Metabolomics 2012;8:133-142.

Schaeffer EL, Forlenza OV, Gattaz WF. Phospholipase A(2) activation as a therapeutic approach for cognitive enhancement in early-stage Alzheimer disease. Psychopharmacology (Berl) 2009;202:37-51.

Schaeffer EL, Gattaz WF. Cholinergic and glutamatergic alterations beginning at the early stages of Alzheimer disease: participation of the phospholipase $A(2)$ enzyme. Psychopharmacology (Berl) 2008;198:1-27.

Selkoe DJ. Alzheimer's disease: Genes, proteins, and therapy. Physiol Rev 2001;81:741-766.

Selkoe DJ. Cell biology of protein misfolding: The examples of Alzheimer's and Parkinson's diseases. Nat Cell Biol 2004;6:1054-1061.

Selkoe DJ, Schenk D. Alzheimer's disease: Molecular understanding predicts amyloid-based therapeutics. Annu Rev Pharmacol Toxicol 2003;43:545-584.

Skovronsky DM, Lee VMY, Trojanowskiz JQ: Neurodegenerative diseases: New concepts of pathogenesis and their therapeutic implications; in: Annual Review of PathologyMechanisms of Disease. Annual Review of Pathology-Mechanisms of Disease. 2006, vol 1, pp 151-170.

Stephenson DT, Lemere CA, Selkoe DJ, Clemens JA. Cytosolic phospholipase A(2) (cPLA(2)) immunoreactivity is elevated in Alzheimer's disease brain. Neurobiol Dis 1996;3:51-63.

Trushina E, Dutta T, Persson X-MT, Mielke MM, Petersen RC. Identification of altered metabolic pathways in plasma and CSF in Mild Cognitive Impairment and Alzheimer's disease using metabolomics. PLoS One 2013;8.

Trushina E, Nemutlu E, Zhang S, Christensen T, Camp J, Mesa J, Siddiqui A, Tamura Y, Sesaki H, Wengenack TM, Dzeja PP, Poduslo JF. Defects in Mitochondrial Dynamics and Metabolomic Signatures of Evolving Energetic Stress in Mouse Models of Familial Alzheimer's Disease. PLoS One 2012;7. 
Urban M, Enot DP, Dallmann G, Korner L, Forcher V, Enoh P, Koal T, Keller, Deigner HP. Complexity and pitfalls of mass spectrometry-based targeted metabolomics in brain research. Analytical Biochemistry 2010;406:124-131.

Volianskis A, Kostner R, Molgaard M, Hass S, Jensen MS. Episodic memory deficits are not related to altered glutamatergic synaptic transmission and plasticity in the CA1 hippocampus of the APPswe/PS1 Delta E9-deleted transgenic mice model of betaamyloidosis. Neurobiol Aging 2010;31:1173-1187.

Xia J, Mandal R, Sinelnikov IV, Broadhurst D, Wishart DS. MetaboAnalyst 2.0-A comprehensive server for metabolomic data analysis. Nucleic Acids Res 2012;40:W127-W133.

Xiong H, Callaghan D, Wodzinska J, Xu J, Premyslova M, Liu Q-Y, Connelly J, Zhang W. Biochemical and behavioral characterization of the double transgenic mouse model (APPswe/PS1dE9) of Alzheimer's disease. Neurosci Bull 2011;27:221-232.

Yao JK, Wengenack TM, Curran GL, Poduslo JF. Reduced membrane lipids in the cortex of Alzheimer's disease transgenic mice. Neurochem Res 2009;34:102-108.

Yatin SM, Yatin M, Aulick T, Ain KB, Butterfield DA. Alzheimer's amyloid beta-peptide associated free radicals increase rat embryonic neuronal polyamine uptake and ornithine decarboxylase activity: protective effect of vitamin E. Neurosci Lett 1999;263:17-20.

Yatin SM, Yatin M, Varadarajan S, Ain KB, Butterfield DA. Role of spermine in amyloid betapeptide-associated free radical-induced neurotoxicity. J Neurosci Res 2001;63:395401.

Yudkoff M. Brain metabolism of branched-chain amino acids. Glia 1997;21:92-98. 
Table 1 - Overview of Brain and Plasma metabolites significantly altered in APP/PS1 mice

\begin{tabular}{|c|c|c|c|c|c|c|c|c|c|c|}
\hline Age (months) & \multicolumn{2}{|c|}{6} & \multicolumn{2}{|c|}{8} & \multicolumn{2}{|c|}{10} & \multicolumn{2}{|c|}{12} & \multicolumn{2}{|c|}{18} \\
\hline Sample type & $\begin{array}{l}\text { Brain } \\
(n=9)\end{array}$ & $\begin{array}{c}\text { Plasma } \\
(n=9)\end{array}$ & $\begin{array}{l}\text { Brain } \\
(\mathrm{n}=8)\end{array}$ & $\begin{array}{c}\text { Plasma } \\
(n=8)\end{array}$ & $\begin{array}{l}\text { Brain } \\
(\mathrm{n}=8)\end{array}$ & $\begin{array}{c}\text { Plasma } \\
(\mathrm{n}=8)\end{array}$ & $\begin{array}{l}\text { Brain } \\
(\mathrm{n}=8)\end{array}$ & $\begin{array}{c}\text { Plasma } \\
(n=8)\end{array}$ & $\begin{array}{l}\text { Brain } \\
(n=8)\end{array}$ & $\begin{array}{c}\text { Plasma } \\
(n=8)\end{array}$ \\
\hline Amino acids and biogenic amines (42) & $3[7 \%]$ & $3[7 \%]$ & $2[5 \%]$ & $2[5 \%]$ & $4[10 \%]$ & $12[29 \%]$ & $1[2 \%]$ & $6[14 \%]$ & $1[2 \%]$ & $0[0 \%]$ \\
\hline Acylcarnitines (40) & $0[0 \%]$ & $0[0 \%]$ & 3 [8\%] & $6[15 \%]$ & $2[5 \%]$ & $2[5 \%]$ & $1[3 \%]$ & $2[5 \%]$ & $5[13 \%]$ & 6 [15\%] \\
\hline Phosphotidylcholines (PCs) (73) & $0[0 \%]$ & $0[0 \%]$ & 28 [38\%] & $0[0 \%]$ & $1[1 \%]$ & $0[0 \%]$ & $1[1 \%]$ & 51 [70\%] & $0[0 \%]$ & $1[1 \%]$ \\
\hline Lyso-phosphotidylcholines (LysoPCs) (17) & $1[6 \%]$ & $1[6 \%]$ & $2[12 \%]$ & $1[6 \%]$ & $0[0 \%]$ & $1[6 \%]$ & $1[6 \%]$ & 7 [41\%] & $3[18 \%]$ & $1[6 \%]$ \\
\hline Sphingolipids (SPHs) (15) & $1[7 \%]$ & $0[0 \%]$ & 6 [40\%] & $0[0 \%]$ & $0[0 \%]$ & $0[0 \%]$ & 3 [20\%] & 3 [20\%] & $1[7 \%]$ & $0[0 \%]$ \\
\hline Total (187) & $5[3 \%]$ & $4[2 \%]$ & 41 [22\%] & 9 [5\%] & $7[4 \%]$ & $15[8 \%]$ & $7[4 \%]$ & 69 [37\%] & 10 [19\%] & 8 [4\%] \\
\hline $\begin{array}{l}\text { Ability of OPLS-DA to predict class } \\
\text { membership (Q2 cummulative) }\end{array}$ & 0.174 & 0.406 & 0.836 & 0.581 & 0.701 & 0.837 & 0.701 & 0.890 & 0.387 & 0.697 \\
\hline
\end{tabular}

Numbers in parenthesis indicate the total number of metabolites measured in each metabolite class. Number of metabolites refer to those significantly different in APP/PS1 mice compared to WT (Kruskal-Wallis one-way ANOVA with pairwise multiple comparisons). Values in square brackets are the respective percentage of measured metabolites altered. Q2 values from OPLS-DA are cumulative. 
Table 2 - Significant alternations in amino acid and biogenic amine concentrations.

\begin{tabular}{|c|c|c|c|}
\hline & $\begin{array}{c}\text { Age } \\
\text { (months) }\end{array}$ & $\begin{array}{l}\% \text { change in } \\
\text { APP/PS1 mice }\end{array}$ & $p$-value \\
\hline \multicolumn{4}{|c|}{ Brain } \\
\hline Phenylalanine & 6 & $+20.11 \pm 26.38$ & 0.013 \\
\hline \multirow{2}{*}{ Trypotophan } & 6 & $+30.53 \pm 40.54$ & 0.021 \\
\hline & 18 & $+33.06 \pm 41.34$ & 0.025 \\
\hline Tyrosine & 12 & $-27.92 \pm 20.17$ & 0.034 \\
\hline \multicolumn{4}{|c|}{ Plasma } \\
\hline$\alpha$-Aminoadipic acid & 12 & $-68.17 \pm 4.52$ & 0.001 \\
\hline Asparagine & 10 & $+37.96 \pm 30.90$ & 0.007 \\
\hline Histidine & 10 & $+15.91 \pm 10.96$ & 0.036 \\
\hline Phenylalanine & 10 & $+17.92 \pm 8.04$ & 0.002 \\
\hline Valine & 12 & $-0.99 \pm 9.05$ & 0.039 \\
\hline Isoleucine & 12 & $+14.98 \pm 12.68$ & 0.003 \\
\hline \multirow{2}{*}{ Methionine } & 10 & $+35.91 \pm 39.90$ & 0.015 \\
\hline & 12 & $-33.67 \pm 13.17$ & 0.003 \\
\hline \multirow{2}{*}{ Tyrosine } & 8 & $+31.73 \pm 27.98$ & 0.015 \\
\hline & 10 & $+30.02 \pm 14.27$ & 0.004 \\
\hline \multirow{2}{*}{ Methionine sulfoxide } & 6 & $+23.97 \pm 23.72$ & 0.025 \\
\hline & 10 & $+73.13 \pm 97.81$ & 0.011 \\
\hline Serotonin & 8 & $+72.72 \pm 39.24$ & 0.027 \\
\hline \multirow{2}{*}{ Taurine } & 6 & $+17.99 \pm 16.09$ & 0.034 \\
\hline & 10 & $+27.85 \pm 15.52$ & 0.003 \\
\hline
\end{tabular}

Changes in APP/PS1 mice are expressed as percentages compared with WT. ' +' indicates an increase and '-' a decrease. P-values were calculated using the Kruskal-Wallis one-way ANOVA with pairwise multiple comparisons. Data are presented as mean $\pm S D(n=8-9)$. 
Figure Legends

Figure 1. Multivariate statistical models arising from targeted metabolomics data. OPLS-DA scores plot classifying the brain samples (upper) and plasma samples (lower) from WT (circle) and APP/PS1 (triangle) mice aged (blue) 6 months, (red) 8 months, (green) 10 months, (yellow) 12 months and (grey) 18 months.

Figure 2. Age-dependent changes in metabolic pathways in brain samples. Data are presented as mean \pm SD $(n=8-9)$. Kruskal-Wallis one-way ANOVA with pairwise multiple comparisons showed significant differences between WT (black) and APP/PS1 (red) mice. ${ }^{*} \mathrm{p}<0.05$ and ${ }^{* *} \mathrm{p}<0.01$.

Figure 3. Age-dependent changes in metabolic pathways in blood plasma samples. Data are presented as mean \pm SD $(n=8-9)$. Kruskal-Wallis one-way ANOVA with pairwise multiple comparisons showed significant differences between WT (black) and APP/PS1 (red) mice. ${ }^{*} p<0.05,{ }^{* *} p<0.01$ and ${ }^{* * *} p<0.001$.

Figure 4. Significant changes in acylcarnitine concentrations. Graphs show changes in individual acylcarnitines in brain (upper) and plasma (lower) of the APP/PS1 mice relative to WT mice $(6,8,10,12$ and 18 months). Data are presented as mean $\pm S D(n=8-9)$. Statistical significances were determined using the Kruskal-Wallis one-way ANOVA with pairwise multiple comparisons $\left({ }^{*} p<0.05,{ }^{* *} p<0.01\right.$ and $\left.{ }^{* *} p<0.001\right)$.

Figure 5. Phosophlipid disturbances in the APP/PS1 mouse. Heat maps show the changing phospholipid profiles (14 lysophosphatidylcholine (LysoPCs), 76 Phosphatidylcholine (PCs) and 15 sphingomyelin (SPHs)) of WT and APP/PS1 mice. (upper) brain phospholipids; (lower) plasma phospholipds. Each individual row represents a sample, and each individual column represents a phospholipid. Red pixels indicate increasing concentrations and green pixels decreasing concentrations (see colour scale above the heat map). Yellow boxes indicate the divergent changes detected in brain and plasma at 8 and 12 months, respectively. Heat map visualisations were produced with PermutMatrix graphical interface after Z-score normalization and Pearson's dissimilarity was used as distance measure. 
Figure 1

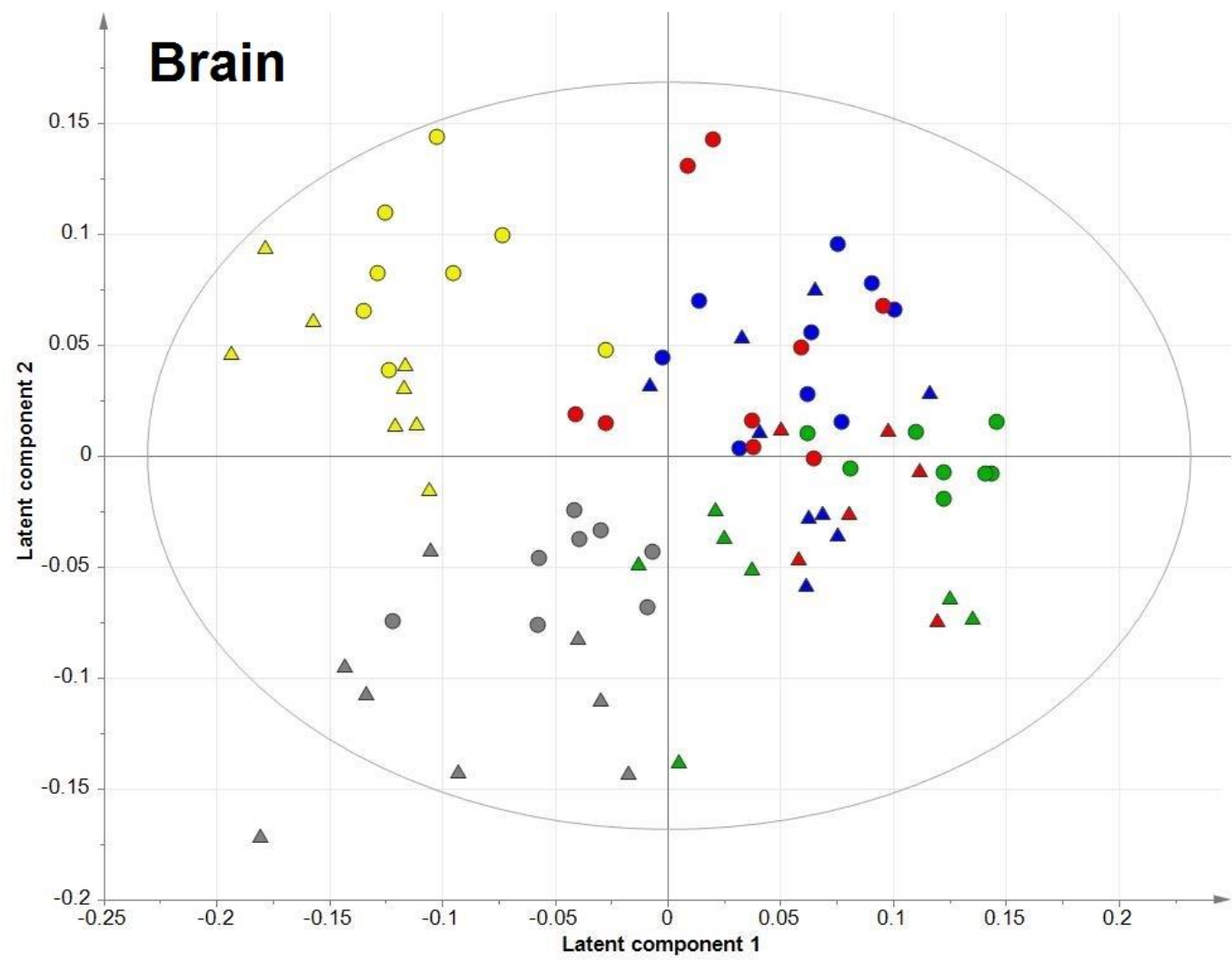

- 6 months WT

A 6 months APP/PS1

8 months WT

8 months APPIPS 1

10 months WT

$\Delta 10$ months APP/PS1

12 months WT

$\triangle 12$ months APP/PS1

18 months WT

$\triangle 18$ months APP/PS1

Latent component 1

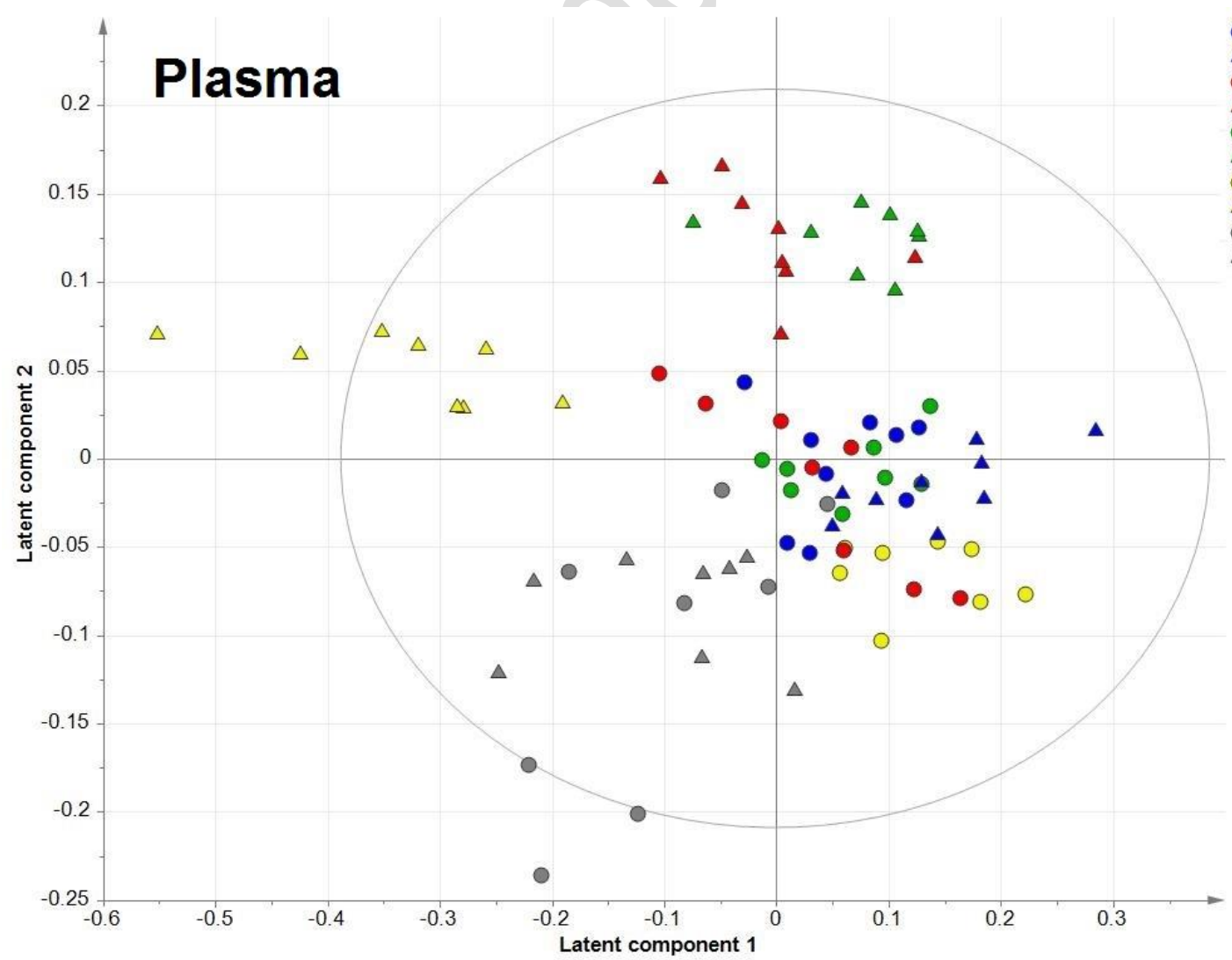

6 months WT

A 6 months APP/PS 1

8 months WT

8 months APP/PS 1

10 months WT

$\triangle 10$ months APP/PS

12 months WT

$\triangle 12$ months APP/PS1

18 months WT

$\triangle 18$ months APP/PS1

Latent component 1 
Figure 2

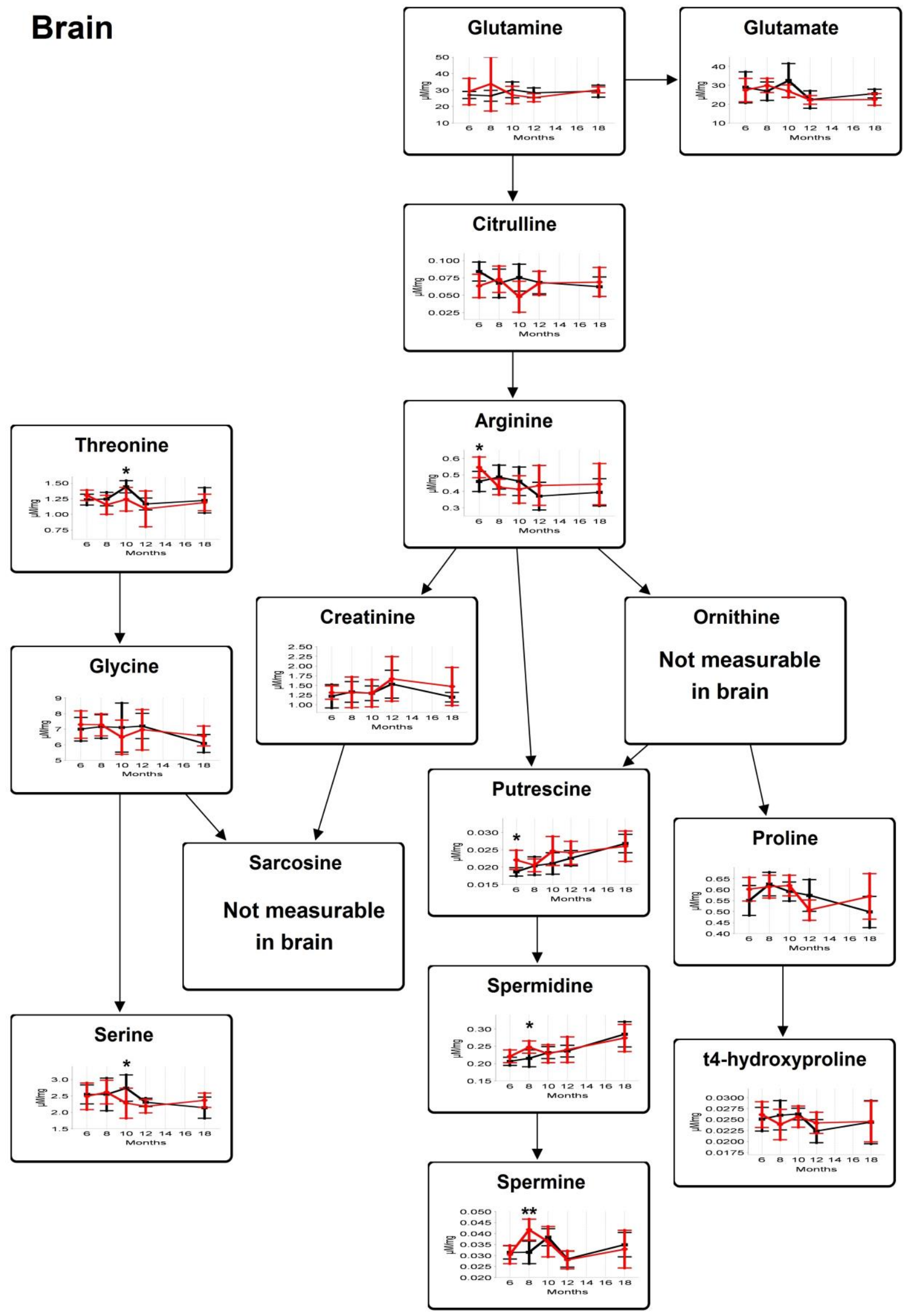


Figure 3

Plasma

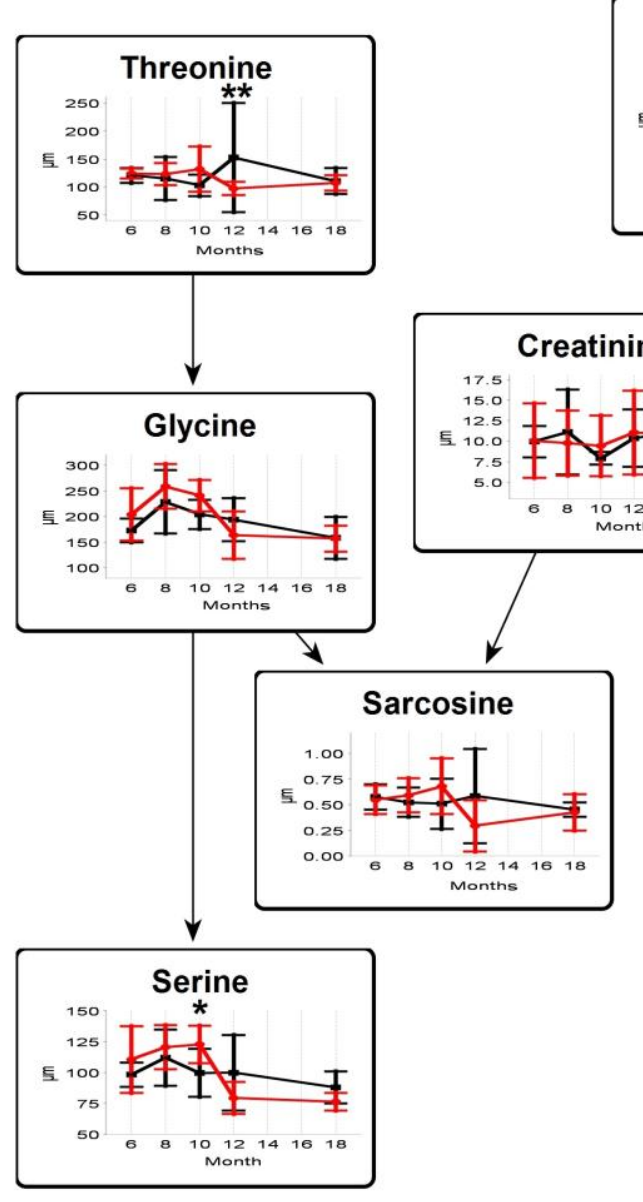

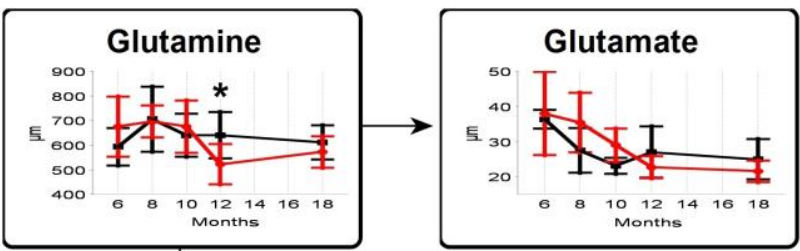

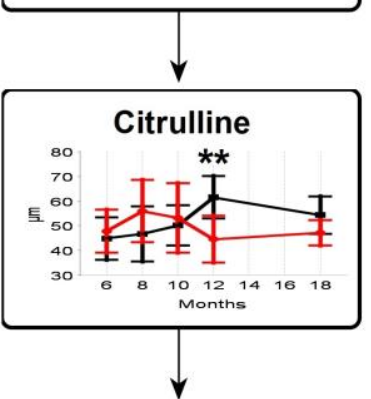

Arginine

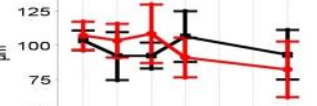

$\begin{array}{lllllllll}50 & 6 & 8 & 10 & 12 & 14 & 16 & 18\end{array}$
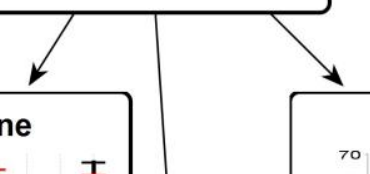

\section{Ornithine}
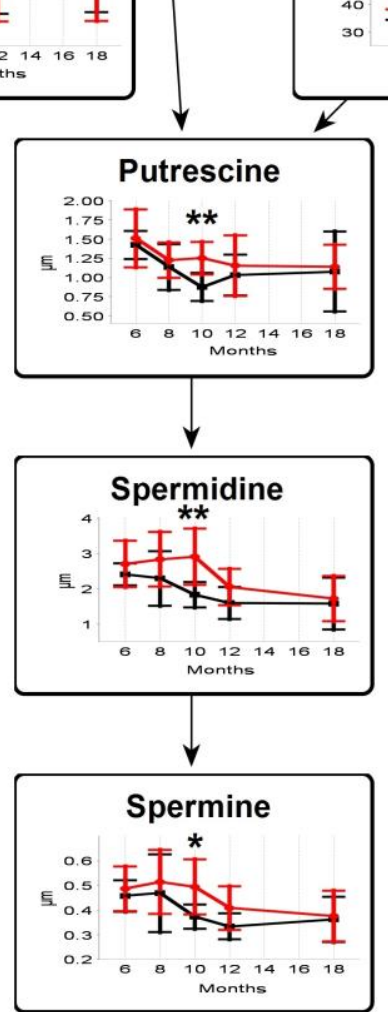
Figure 4
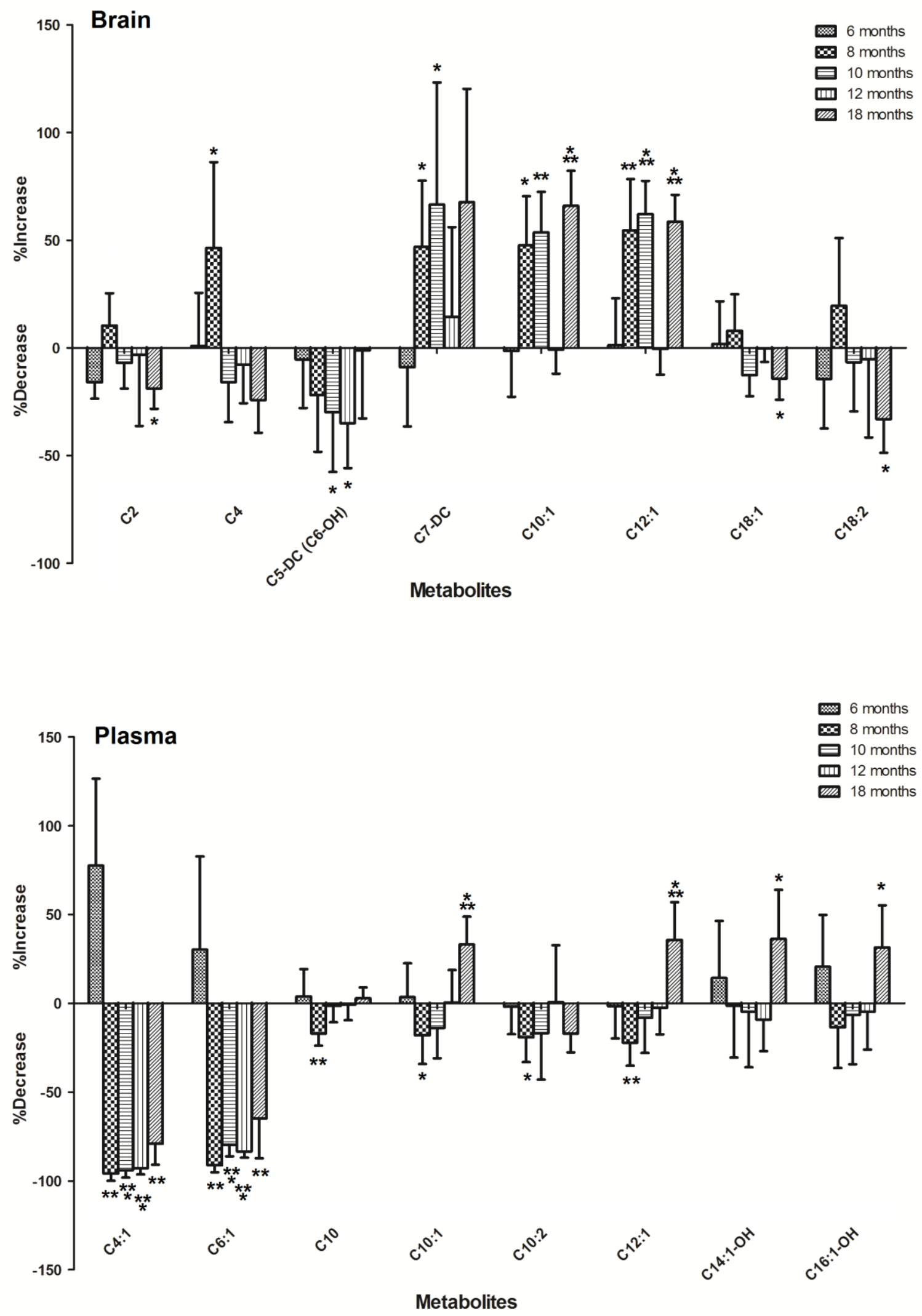
Figure 5

\section{Brain}
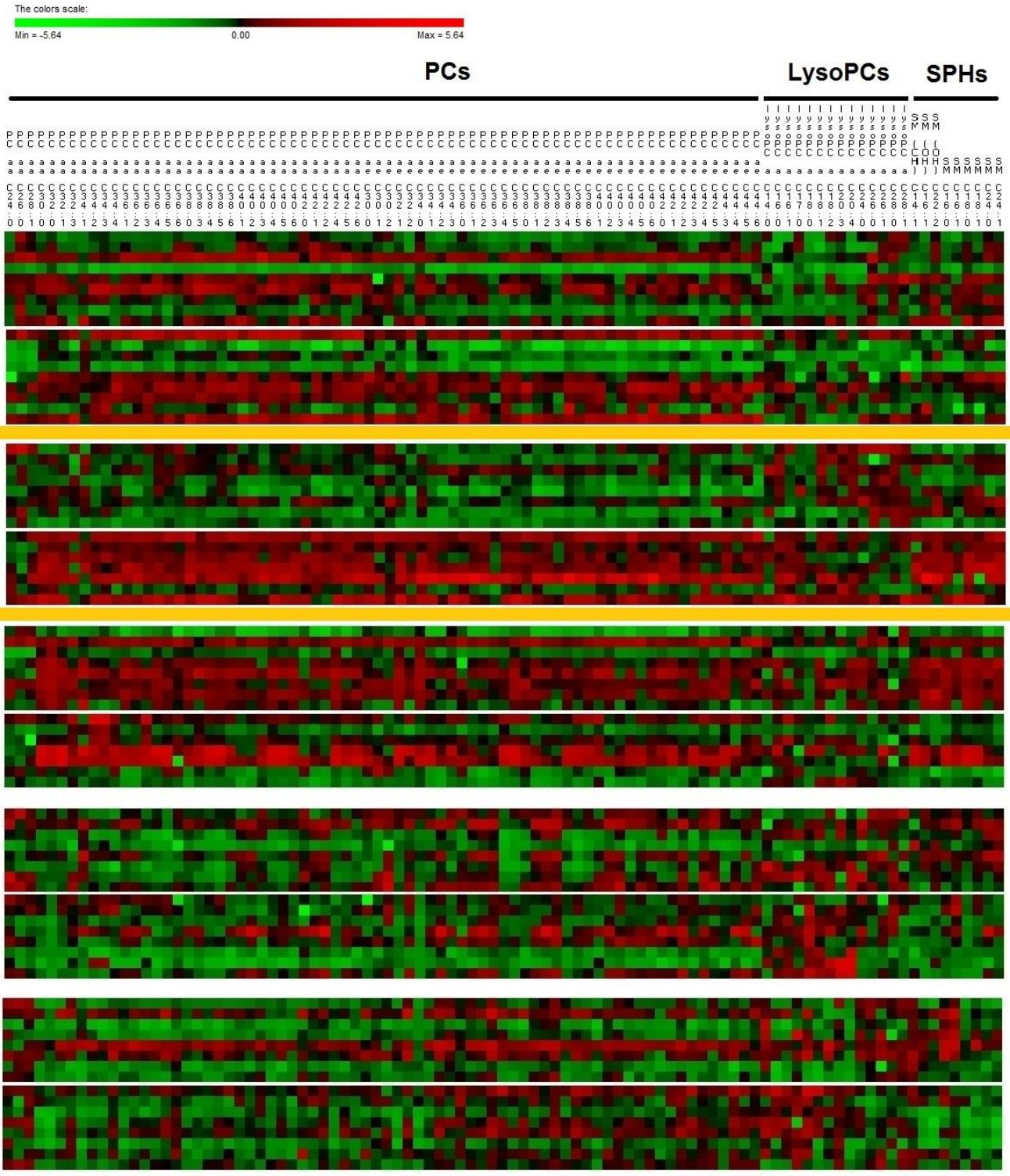

Mouse group

Wild type

APP/PS1

Time point

6 months

8 months

10 months

12 months

18 months 


\section{Plasma}

The colors scale:

$\begin{array}{lll}\text { Min }=-7.28 & 0.00 & \end{array}$

PCs

LysoPCs SPHs

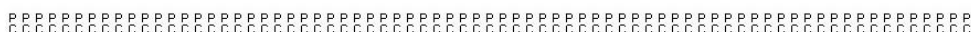

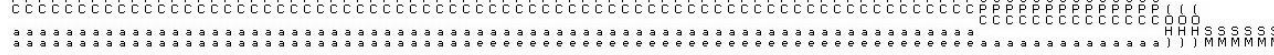

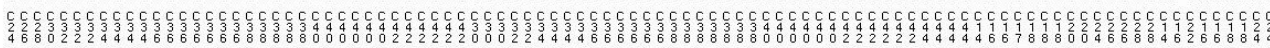
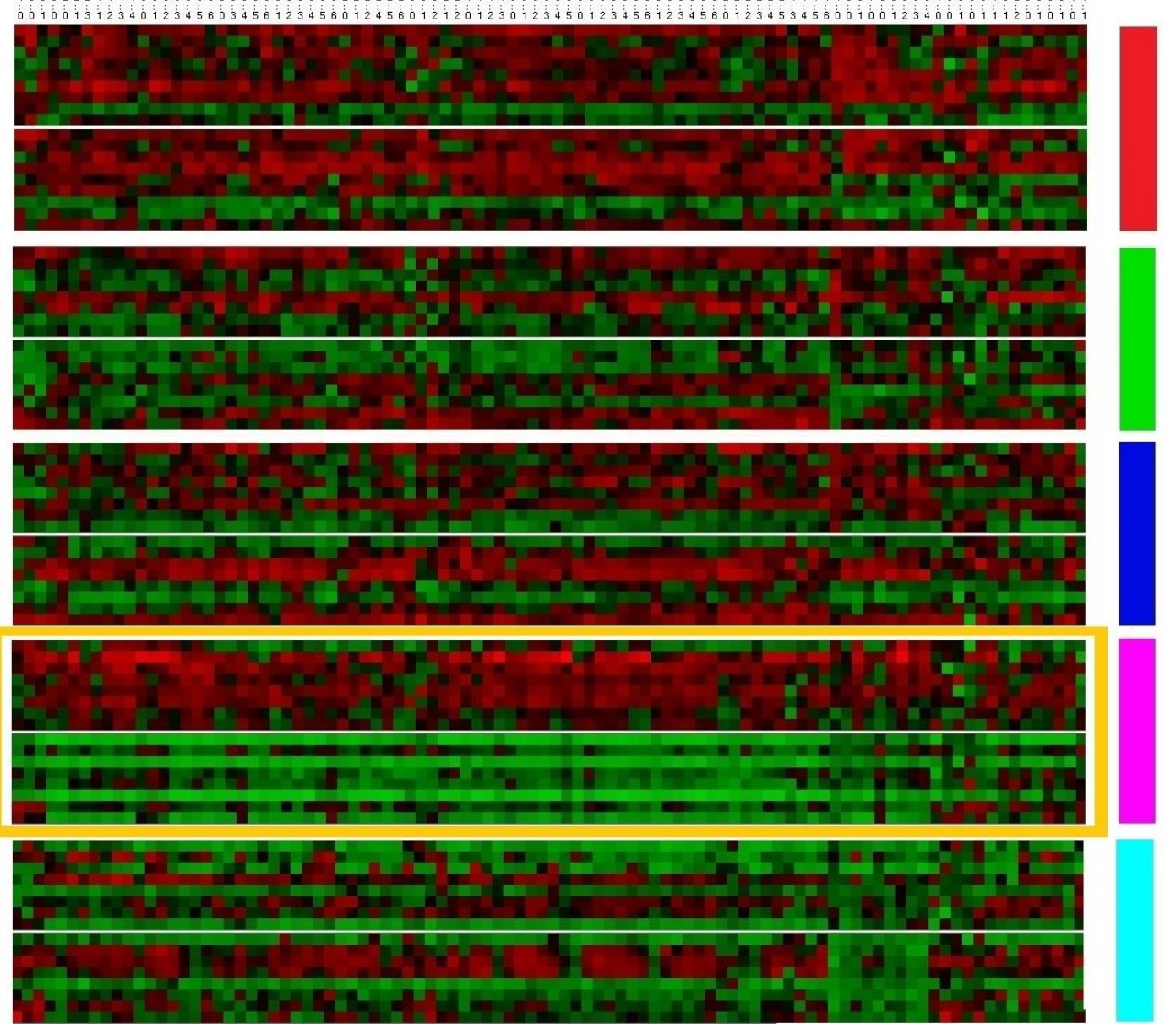

Mouse group

Wild type

APP/PS1

Time point

6 months

8 months

10 months

12 months

18 months 
Supplementary Table 1 - Alterations in essential and branched chain amino acids in APP/PS1 mice.

\begin{tabular}{|c|c|c|c|c|c|}
\hline \multicolumn{2}{|c|}{} & \multicolumn{2}{|c}{ EAA:NEAAs } & \multicolumn{3}{c|}{ BCAAs } \\
\hline \multirow{4}{*}{ Brain } & $\mathbf{6}$ months & $0.049 \pm 0.010$ & $0.050 \pm 0.005$ & $1.49 \pm 0.40$ & $1.46 \pm 0.51$ \\
\cline { 2 - 6 } & $\mathbf{8}$ months & $0.051 \pm 0.005$ & $0.048 \pm 0.006$ & $1.56 \pm 0.20$ & $1.57 \pm 0.38$ \\
\cline { 2 - 6 } & $\mathbf{1 0}$ months & $0.046 \pm 0.005$ & $0.051 \pm 0.004$ & $1.31 \pm 0.10$ & $1.55 \pm 0.30$ \\
\cline { 2 - 6 } & $\mathbf{1 2}$ months & $0.048 \pm 0.009$ & $0.049 \pm 0.004$ & $1.29 \pm 0.51$ & $1.24 \pm 0.25$ \\
\cline { 2 - 6 } & $\mathbf{1 8}$ months & $0.046 \pm 0.006$ & $0.051 \pm 0.004$ & $1.16 \pm 0.41$ & $1.47 \pm 0.19$ \\
\hline \multirow{4}{*}{ Plasma } & $\mathbf{6}$ months & $0.53 \pm 0.041$ & $\mathbf{0 . 4 7} \pm \mathbf{0 . 0 5 7}$ & $395.52 \pm 22.90$ & $374.27 \pm 43.63$ \\
\cline { 2 - 6 } & $\mathbf{8}$ months & $0.46 \pm 0.039$ & $0.45 \pm 0.036$ & $377.89 \pm 37.42$ & $381.54 \pm 50.55$ \\
\cline { 2 - 6 } & $\mathbf{1 0}$ months & $0.46 \pm 0.053$ & $0.47 \pm 0.079$ & $343.59 \pm 40.77$ & $394.31 \pm 86.16$ \\
\cline { 2 - 6 } & $\mathbf{1 2}$ months & $0.48 \pm 0.122$ & $\mathbf{0 . 5 2} \pm \mathbf{0 . 0 4 2}{ }^{* *}$ & $328.01 \pm 138.91$ & $331.90 \pm 29.48$ \\
\cline { 2 - 6 } & $\mathbf{1 8}$ months & $0.46 \pm 0.022$ & $\mathbf{0 . 5 2} \pm \mathbf{0 . 0 4 7}{ }^{*}$ & $307.55 \pm 44.85$ & $338.32 \pm 57.72$ \\
\hline
\end{tabular}

Data are presented as mean \pm SD. EAA:NEAAs - ratio of essential amino acids to non-essential amino acids; BCAAs - Branched chain amino acids. Brain concentrations values are stated in $\mu \mathrm{M} / \mathrm{mg}$ tissue. Plasma concentrations values are stated in $\mu \mathrm{M}$. Statistical differences were determined using the Kruskal-Wallis one-way ANOVA with pairwise multiple comparisons $\left({ }^{*} \mathrm{p}<0.05,{ }^{* *} \mathrm{p}<0.01\right)$. 


\section{Supplementary Table 2 - Alterations in total phosphotidylcholine (PC), Lyso-PC, and sphingomyelin (SPH) in APP/PS1}

mice.

\begin{tabular}{|c|c|c|c|c|c|c|c|}
\hline & \multicolumn{2}{|c|}{ Total PC } & \multicolumn{2}{|c|}{ Total LysoPC } & \multicolumn{2}{|c|}{ Total SPH } \\
\hline & & WT & APP/PS1 & WT & APP/PS1 & WT & APP/PS1 \\
\hline \multirow{5}{*}{ Brain } & 6 months & $85.40 \pm 8.98$ & $85.20 \pm 5.56$ & $0.83 \pm 0.10$ & $0.94 \pm 0.11$ & $4.73 \pm 0.64$ & $4.35 \pm 0.62$ \\
\hline & 8 months & $81.34 \pm 5.63$ & $92.04 \pm 6.38^{*}$ & $1.01 \pm 0.10$ & $1.07 \pm 0.08$ & $4.24 \pm 0.71$ & $5.55 \pm 0.55^{\star \star}$ \\
\hline & 10 months & $90.21 \pm 9.08$ & $87.03 \pm 13.14$ & $0.99 \pm 0.11$ & $1.00 \pm 0.10$ & $5.13 \pm 0.63$ & $4.58 \pm 1.27$ \\
\hline & 12 months & $83.07 \pm 5.63$ & $77.46 \pm 5.32$ & $1.00 \pm 0.12$ & $1.06 \pm 0.09$ & $4.30 \pm 0.38$ & $3.74 \pm 0.22$ \\
\hline & 18 months & $78.70 \pm 7.83$ & $74.91 \pm 4.94$ & $0.88 \pm 0.13$ & $1.02 \pm 0.16$ & $3.93 \pm 0.48$ & $3.45 \pm 0.70$ \\
\hline \multirow{5}{*}{ Plasma } & 6 months & $1487.56 \pm 214.01$ & $1411.09 \pm 254.60$ & $598.94 \pm 81.70$ & $566.99 \pm 125.68$ & $43.80 \pm 11.33$ & $41.75 \pm 7.55$ \\
\hline & 8 months & $1344.11 \pm 184.52$ & $1205.80 \pm 134.43$ & $563.42 \pm 57.73$ & $513.28 \pm 54.41$ & $46.58 \pm 9.68$ & $42.73 \pm 10.07$ \\
\hline & 10 months & $1302.13 \pm 138.85$ & $1319.71 \pm 280.00$ & $564.61 \pm 48.57$ & $551.00 \pm 114.82$ & $41.22 \pm 5.97$ & $40.37 \pm 6.40$ \\
\hline & 12 months & $1544.65 \pm 330.72$ & $996.40 \pm 330.72^{\star \star}$ & $573.37 \pm 62.78$ & $449.01 \pm 62.78^{\star \star}$ & $40.57 \pm 9.49$ & $38.10 \pm 9.49$ \\
\hline & 18 months & $1243.58 \pm 253.09$ & $1308.20 \pm 220.65$ & $452.41 \pm 55.19$ & $440.59 \pm 28.09$ & $40.52 \pm 6.60$ & $40.41 \pm 9.42$ \\
\hline
\end{tabular}

Data are presented as mean \pm SD. Brain concentrations values are stated in $\mu \mathrm{M} / \mathrm{mg}$ tissue. Plasma concentrations values are stated in $\mu \mathrm{M}$. Statistical differences were determined using the Kruskal-Wallis one-way ANOVA with pairwise multiple comparisons $\left({ }^{*} p<0.05,{ }^{* *} p<0.01\right)$. 